\title{
Bilateral Macular Edema in a Patient Treated with Tamoxifen: A Case Report and Review of the Literature
}

\author{
Paraskevas Zafeiropoulos $^{\mathrm{a}}$ Panagiotis Nanos $^{\mathrm{b}} \quad$ Evangelos Tsigkoulis $^{\mathrm{a}}$ \\ Maria Stefaniotou ${ }^{\mathrm{a}}$ \\ ${ }^{a}$ University Ophthalmology Clinic of Ioannina, Ioannina, and ${ }^{b}$ University Eye Clinic, \\ Democritus University of Thrace, Alexandroupolis, Greece
}

\section{Key Words}

Tamoxifen retinopathy $\cdot$ Tamoxifen therapy $\cdot$ Macular edema

\begin{abstract}
We present a case of a 41-year-old female patient with progressive bilateral visual loss. On examination, her best corrected visual acuity (BCVA) in her right eye was $3 / 10$ and her BCVA in her left eye was $2 / 10$. Fundus and optical coherence tomography examination revealed severe bilateral macular edema. She had been diagnosed with breast cancer 6 years ago and was receiving tamoxifen at a dosage of $20 \mathrm{mg} /$ day ever since. Tamoxifen therapy was discontinued, and the patient received $250 \mathrm{mg}$ of acetazolamide three times a day for a period of 1 month. Both foveae regained their normal contour within 2 months, and her vision was restored to 10/10 BCVA 3 months later. To our knowledge, this is the first case reported where bilateral intraretinal macular edema is the only retinal manifestation in a patient on oral tamoxifen.

(c) 2014 S. Karger AG, Basel
\end{abstract}

\section{Introduction}

Tamoxifen is an antiestrogenic drug used frequently as a coadjutant treatment in breast cancer. Ocular complications of tamoxifen are rare and include vortex keratopathy, bilateral crystalline maculopathy, macular edema and optic neuritis [1-4]. We report a patient who developed bilateral macular edema without crystalline deposits while receiving tamoxifen. Maculopathy secondary to tamoxifen therapy was considered. 
Zafeiropoulos et al.: Bilateral Macular Edema in a Patient Treated with Tamoxifen: A Case Report and Review of the Literature

\section{Case Report}

A 41-year-old female patient was referred to our department for progressive bilateral visual loss over the last 2 months. She had been diagnosed with breast cancer and had undergone a radical mastectomy 6 years ago. Since then, she had been receiving tamoxifen at a dosage of $20 \mathrm{mg} /$ day.

On examination, the Snellen best corrected visual acuity (BCVA) in her right eye (OD) was $3 / 10$ and that in her left eye (OS) was $2 / 10$. Intraocular pressure was normal and anterior segment examination was unremarkable. Fundus examination revealed severe bilateral macular edema. Optical coherence tomography (OCT) scan showed bilateral, intraretinal macular edema with a central macular thickness of $506 \mu \mathrm{m}$ in her OD and of 469 $\mu \mathrm{m}$ in her OS, respectively (fig. 1). Fluorescein angiography showed late-phase diffuse hyperfluorescence corresponding to the edematous area in both eyes (fig. 2). A diagnosis of central serous retinopathy was excluded because fluorescein angiography revealed no leakage point from the choroid in early phases. Furthermore, no pigment epithelium detachment was detected.

Due to a history of tamoxifen administration for 6 years and absence of other pathologies causing macular edema, maculopathy secondary to tamoxifen therapy was considered. Tamoxifen therapy was discontinued. Instead of tamoxifen, the patient was administered $1 \mathrm{mg}$ anastrozole daily which is a nonsteroidal aromatase inhibitor. The patient received $250 \mathrm{mg}$ of acetazolamide three times a day and nepafenac drops three times a day to both eyes for a period of 1 month.

Shortly after this, the central macular thickness started to diminish with both foveae regaining their normal contour within 2 months (fig. 3). Subsequently, her vision was restored to 10/10 BCVA 3 months later.

\section{Discussion}

Tamoxifen-induced retinopathy is a rare complication. The incidence among patients receiving tamoxifen is $0.6 \%$ and can increase up to $10.9 \%$ with the use of chemotherapy. The first case of tamoxifen-induced retinopathy was first described by Kaiser-Kupfer and Lippman in 1978 (it was about a woman who received extremely high doses of tamoxifen due to metastatic breast cancer) [1]. Since then, a number of studies have suggested that the use of low doses (20-40 mg/day) may be associated with a decrease in visual acuity and tamoxifen-induced retinopathy.

There are two types of tamoxifen-induced retinopathy. One of them is an acute form, which manifests as diminished vision, retinal edema, retinal hemorrhage and optic disk swelling. This form may be due to the estrogenic activity of tamoxifen and is reversible after the discontinuation of the drug [5]. Selective estrogen receptor modulators such as tamoxifen interact with two kinds of estrogenic receptors (ERs) referred to as $\alpha$ and $\beta$. The ratio of $E R \alpha$ and $E R \beta$ to a tissue is associated with the function of selective estrogen receptor modulators as estrogenic or antiestrogenic [6].

After a long-term use of the drug, typical tamoxifen-induced retinopathy is characterized by the presence of crystalline maculopathy, which consists of refractive intraretinal crystalline deposits, especially in the perifoveal area that histologically may represent the products of axonal degeneration [2]. This damage might explain the associated visual loss caused by this type of retinopathy that may not reverse when tamoxifen is discontinued. 
Zafeiropoulos et al.: Bilateral Macular Edema in a Patient Treated with Tamoxifen: A Case Report and Review of the Literature

Tamoxifen maculopathy is also often associated with macular edema, which is an accumulation of intraretinal fluid that subsequently decreases visual acuity.

More recent studies with 3D-OCT reveal intraretinal cysts, even in patients with no visual symptoms and normal fundoscopy. Therefore 3D-OCT screening is recommended for all patients treated with tamoxifen $[3,4,7,8]$.

In our case, discontinuation of the drug improved vision. We also administer oral acetazolamide and nepafenac eyedrops. Carbonic anhydrase inhibitors increase fluid transport across the retinal pigment epithelium by inhibition of carbonic anhydrase. They are effective in cystoid macular edema and may also be effective in other types of macular edema. Nonsteroidal anti-inflammatory drugs such as nepafenac inhibit the enzyme cyclooxygenase, which is required for the production of the prostaglandins and may be beneficial in managing retinal edema [9].

In our case, there were severe bilateral intraretinal edemas documented by OCT without the presence of crystalline retinopathy. To our knowledge, this is the first case reported, where bilateral intraretinal macular edema is the only retinal manifestation in a patient on oral tamoxifen.

\section{Conclusion}

Bilateral macular edema can be a manifestation of tamoxifen-induced retinopathy without the presence of typical crystalline deposits. Patients under tamoxifen treatment must periodically be examined by ophthalmologists for the early detection of tamoxifen-induced retinopathy and discontinuation of tamoxifen treatment if needed.

\section{Disclosure Statement}

The authors have not received grant support or research funding, and they do not have any proprietary interests in the material described in the article.

\section{References}

1 Srikantia N, Mukesh S, Krishnaswamy M: Crystalline maculopathy: a rare complication of tamoxifen therapy. J Cancer Res Ther 2010;6:313-315.

2 Nair AG, Das D, Goyal A, Gandhi RA: The eyes have it! Tamoxifen maculopathy revisited: a case report. J Ocul Pharmacol Ther 2012;28:640-642.

3 Baget-Bernaldiz M, Soler Lluis N, Romero-Aroca P, Traveset-Maeso A: Optical coherence tomography study in tamoxifen maculopathy. Arch Soc Esp Oftalmol 2008;83:615-618.

-4 Doshi RR, Fortun JA, Kim BT, Dubovy SR, Rosenfeld PJ: Pseudocystic foveal cavitation in tamoxifen retinopathy. Am J Ophthalmol 2014;157:1291-1298.

5 Li J, Tripathi RC, Tripathi BJ: Drug-induced ocular disorders. Drug Saf 2008;31:127-141.

6 Maximov PY, Lee TM, Jordan VC: The discovery and development of selective estrogen receptor modulators (SERMs) for clinical practice (review). Curr Clin Pharmacol 2013;8:135-155.

7 Chung H, Kim D, Ahn SH, Kim JG, Lee JY, Lim JY, Yoon YH: Early detection of tamoxifen-induced maculopathy in patients with low cumulative doses of tamoxifen. Ophthalmic Surg Lasers Imaging 2010;9:1-5.

-8 Georgalas I, Paraskevopoulos T, Papaconstaninou D, Brouzas D, Koutsandrea C: Large bilateral foveal cysts in the inner retina of a patient treated with tamoxifen, diagnosed with Fourier-domain optical coherence tomography. Clin Ophthalmol 2013;7:707-709.

-9 Rotsos TG, Moschos MM: Cystoid macular edema. Clin Ophthalmol 2008;2:919-930. 
Case Reports in

Ophthalmology

\begin{tabular}{l|l}
\hline Case Rep Ophthalmol 2014;5:451-454 \\
\hline DOI: 10.1159/000370144 & $\begin{array}{l}\text { @ 2014 S. Karger AG, Basel } \\
\text { www.karger.com/cop }\end{array}$ \\
\hline
\end{tabular}

Zafeiropoulos et al.: Bilateral Macular Edema in a Patient Treated with Tamoxifen: A Case Report and Review of the Literature
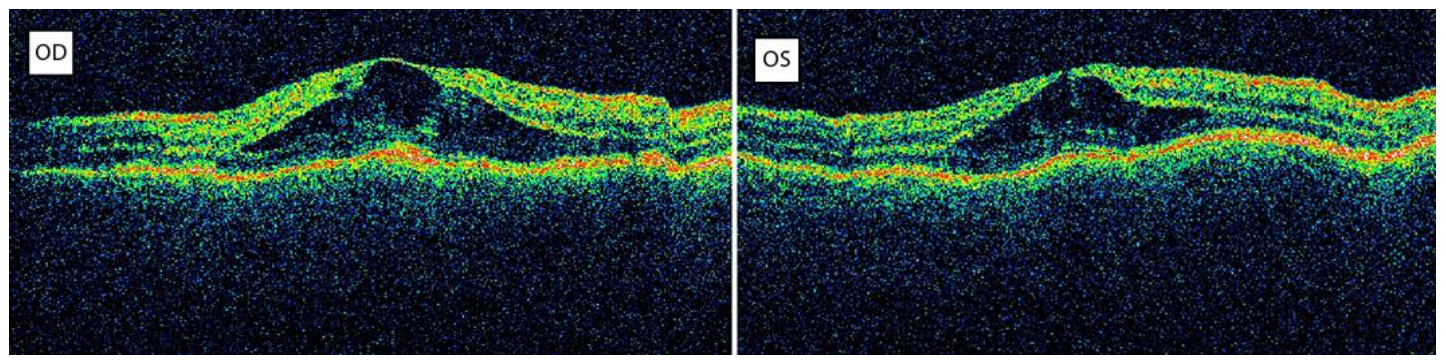

Fig. 1. OCT. Severe bilateral intraretinal macular edema.
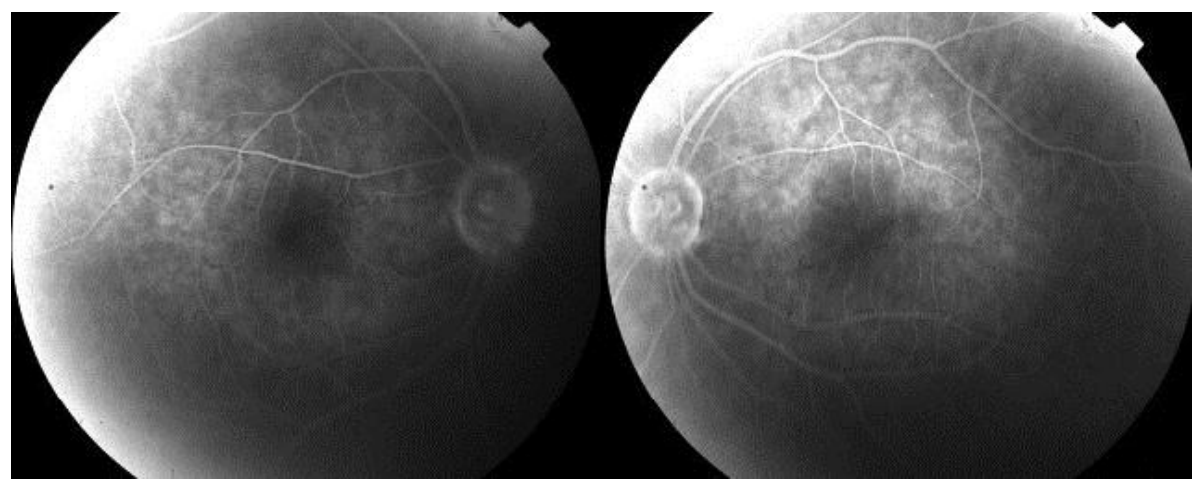

Fig. 2. Fluorescein angiography. Diffuse hyperfluorescene in the late phases of fluorescein angiography.
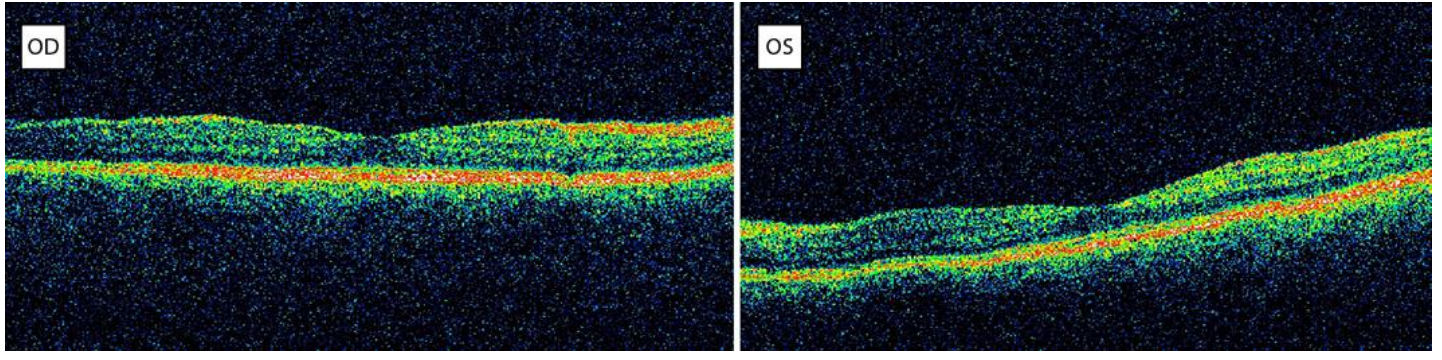

Fig. 3. OCT 2 months after discontinuation of tamoxifen: regression of edema and normal fovea contour bilaterally. 